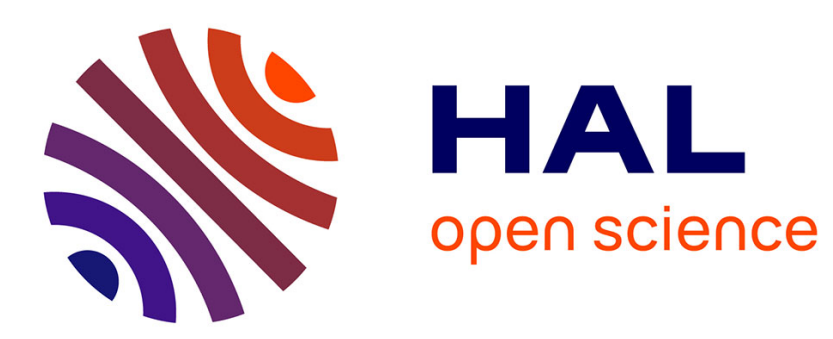

\title{
Calculs d'énergie de faute d'empilement dans le sesquioxyde d'yttrium (Y2O3)
}

\author{
M. Boisson, R.J. Gaboriaud
}

\section{To cite this version:}

M. Boisson, R.J. Gaboriaud. Calculs d'énergie de faute d'empilement dans le sesquioxyde d'yttrium (Y2O3). Journal de Physique Lettres, 1977, 38 (8), pp.177-181. 10.1051/jphyslet:01977003808017700 . jpa-00231354

\section{HAL Id: jpa-00231354 https://hal.science/jpa-00231354}

Submitted on 1 Jan 1977

HAL is a multi-disciplinary open access archive for the deposit and dissemination of scientific research documents, whether they are published or not. The documents may come from teaching and research institutions in France or abroad, or from public or private research centers.
L'archive ouverte pluridisciplinaire HAL, est destinée au dépôt et à la diffusion de documents scientifiques de niveau recherche, publiés ou non, émanant des établissements d'enseignement et de recherche français ou étrangers, des laboratoires publics ou privés. 


\title{
CALCULS D'ÉNERGIE DE FAUTE D'EMPILEMENT DANS LE SESQUIOXYDE D'YTTRIUM $\left(\mathrm{Y}_{2} \mathrm{O}_{3}\right)$
}

\author{
M. BOISSON et R. J. GABORIAUD \\ Laboratoire de Métallurgie Physique, Faculté des Sciences, \\ 40, avenue du Recteur-Pineau, 86022 Poitiers, France
}

(Reçu le 28 janvier 1977, accepté le 10 mars 1977)

\begin{abstract}
Résumé. - Un calcul théorique de l'énergie des différentes fautes d'empilement pouvant être rencontrées dans le sesquioxyde d'yttrium $\left(\mathrm{Y}_{2} \mathrm{O}_{3}\right)$ est proposé.

Le cristal est supposé ionique. Une méthode de sommation d'interactions de paires est employée.

Les énergies de faute d'empilement calculées sont fortes dans les plans (111) et (110) mais plus faibles dans (100).

Une faute d'empilement correspondant au cisaillement $b=a / 2\langle 110\rangle(001)$ de basse énergie $\left(\Gamma=80 \mathrm{~mJ} / \mathrm{m}^{2}\right)$ est trouvée. Une relation est faite avec des résultats expérimentaux de déformation plastique.
\end{abstract}

Abstract. - Theoretical calculations of stacking fault energy have been performed in the yttrium oxide $\left(\mathrm{Y}_{2} \mathrm{O}_{3}\right)$. A method of pair potential interactions, based on an ionic crystal model, is used. The stacking fault energies are found to be high in the (111) and (110) planes but lower in the (100) plane. A low stacking fault energy $\left(\Gamma=80 \mathrm{~mJ} / \mathrm{m}^{2}\right)$ corresponding to the shear $b=a / 2\langle 110\rangle(001)$ is found. This fact is connected to the experimental plastic deformation results obtained in $\mathrm{Y}_{2} \mathrm{O}_{3}$.

1. Introduction. - L'étude des propriétés plastiques des oxydes suscite, depuis quelques années, un regain d'intérêt. Les possibilités, relativement récentes dans ce domaine, d'élaboration de monocristaux, permettent l'étude fondamentale des processus de déformation.

Au même titre que dans les métaux, l'énergie de faute d'empilement (E.F.E.) est un des paramètres pouvant jouer un rôle prépondérant dans les mécanismes de déformation plastique. Malheureusement l'évaluation expérimentale de ce facteur, principalement basée sur la microscopie électronique est difficile et sujette à caution.

Un calcul théorique, donnant un ordre de grandeur de l'E.F.E. et en particulier, montrant une éventuelle différence notable entre plusieurs possibilités de cisaillement est donc d'un intérêt certain.

Dans le cadre de l'étude des propriétés plastiques du sesquioxyde d'yttrium $\left(\mathrm{Y}_{2} \mathrm{O}_{3}\right)$ [1] monocristallin, un calcul d'E.F.E. dans les plans de bas indices a donc été entrepris.

2. Structure. - L'oxyde d'yttrium a une structure cubique type $\mathrm{C}[2,3,4]$ jusqu'à la température de $2350^{\circ} \mathrm{C}$ [5].

Cette structure peut être obtenue à partir de la structure fluorine plus connue $\left(\mathrm{CaF}_{2}, \mathrm{UO}_{2} \ldots\right)$ dans laquelle on enlève le quart des anions de façon particulière.

Le groupe d'espace est $\mathrm{Ia}_{3}$ (Th 7).

Cet oxyde possède 16 molécules d' $\mathrm{Y}_{2} \mathrm{O}_{3}$ par maille, soit 80 atomes.

Le paramètre cristallin est de $a_{0}=10,604 \AA$ à $27^{\circ} \mathrm{C}$.

Une représentation idéalisée de la structure consiste à considérer chaque ion métallique au centre d'un cube entouré de 6 oxygènes. Les 2 oxygènes manquants sont soit sur une diagonale du cube, soit sur une diagonale d'une face créant ainsi 2 sortes de sites pour $\mathrm{Y}^{+++}$appelés respectivement $\mathrm{S}_{6}$ et $\mathrm{C}_{2}$ (Fig. 1).

La structure cubique type $C$ présente donc un sous-réseau anionique largement défectif.

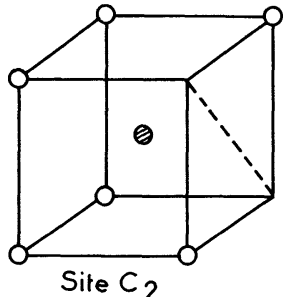

(a)

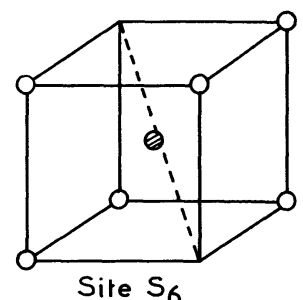

(b)
Fig. 1. - Les sites de $\mathrm{Y}^{+++}$dans $\mathrm{Y}_{2} \mathrm{O}_{3} . a$ ) site $\mathrm{C}_{2} ; b$ ) site $\mathrm{S}_{6}$. [The two $\mathrm{Y}^{+++}$sites in $\left.\mathrm{Y}_{2} \mathrm{O}_{3} . a\right) \mathrm{C}_{2}$ site; b) $\mathrm{S}_{6}$ site.] 
3. Principe du calcul. - Le calcul des E.F.E. basé sur un modèle ionique est réalisé en terme d'interaction de paires.

L'énergie d'interaction entre 2 ions $i$ et $j$ distant de $r_{\mathrm{ij}}$ s'écrit [6] :

$$
\begin{aligned}
W_{\mathrm{ij}}=Z_{\mathrm{i}} Z_{\mathrm{j}} e^{2} r_{\mathrm{ij}}^{-1}-C_{\mathrm{ij}}^{(6)} r_{\mathrm{ij}}^{-6} & -C_{\mathrm{ij}}^{(8)} r_{\mathrm{ij}}^{-8}+ \\
& +b b_{\mathrm{i}} b_{\mathrm{j}} f_{\mathrm{ij}} \exp -\frac{r_{\mathrm{ij}}}{\rho} .
\end{aligned}
$$

On reconnaît dans cette expression

- l'énergie électrostatique ;

- les termes de Van der Waals correspondant aux interactions dipôles-dipôles, dipôles-quadrupôles ;

- l'énergie de répulsion.

Le calcul se résume en termes de sommation d'interactions plan à plan [7]. Le calcul de $W_{\mathrm{ij}}$ défini précédemment nécessite la connaissance d'un certain nombre de paramètres, pour l'évaluation de toutes les composantes. $\mathrm{La}$ fluorine $\mathrm{CaF}_{2}$, par exemple, se prête bien à ce genre de calcul $[8,9]$.

Les données expérimentales disponibles pour $\mathrm{Y}_{2} \mathrm{O}_{3}$ ne permettent que le calcul de la composante électrostatique. Une étude géométrique des liaisons au niveau de la faute permet de prévoir l'importance relative des autres composantes comme la répulsion ou la polarisation et donc d'avoir une idée de la validité des résultats.

4. Calculs d'énergies de faute d'empilement. 4.1 Plans (001). - Les résultats expérimentaux de déformation plastique [1] montrent que les plans (001) jouent un rôle prépondérant dans les mécanismes de déformation plastique. Il y sera donc porté une attention toute particulière.

La séquence complète suivant (100) se compose de 4 plans d'anions notés $A_{1}, A_{2}, A_{3}, A_{4}$ séparés par 4 plans de cations appelés $\alpha$ et $\beta$

$$
\begin{array}{l:lllllllll}
\beta & \mathrm{A}_{1} & \propto & \mathrm{A}_{2} & \beta & \mathrm{A}_{3} & \alpha & \mathrm{A}_{4} & \beta & \mathrm{A}_{1}
\end{array}
$$

les deux couches de cations se déduisent l'une de l'autre par une translation $a / 4\langle 110\rangle$; les couches d'anions sont superposables deux à deux de la façon suivante :

$$
\text { translation } \begin{array}{ll}
\frac{a}{2}\langle 1,0,0\rangle & \mathrm{A}_{1} \leftrightarrow \mathrm{A}_{4} \\
& \mathrm{~A}_{2} \leftrightarrow \mathrm{A}_{3} \\
\frac{a}{2}\langle 0,1,0\rangle & \mathrm{A}_{1} \leftrightarrow \mathrm{A}_{2} \\
& \mathrm{~A}_{3} \leftrightarrow \mathrm{A}_{4} \\
\frac{a}{2}\langle 1,1,0\rangle & \mathrm{A}_{1} \leftrightarrow \mathrm{A}_{3} \\
& \mathrm{~A}_{2} \leftrightarrow \mathrm{A}_{4}
\end{array}
$$

les couches $\beta$ contiennent uniquement des cations en site $\mathrm{C}_{2}$ (Fig. $1 a$ ) alors que les couches $\alpha$ contiennent des cations alternativement disposés suivant les lignes de sites $\mathrm{C}_{2}$ ou $\mathrm{S}_{6}$ (Fig. 1 $a, b$ ). La figure 2 montre la répartition des lacunes dans les couches d'anions.

On constate, en regardant la figure 2, que les fautes résultantes sont différentes selon que le cisaillement a lieu au niveau d'une couche $\alpha$ ou au niveau d'une couche $\beta$.

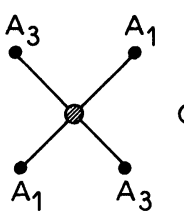

$\otimes$

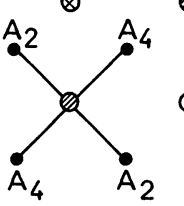

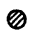

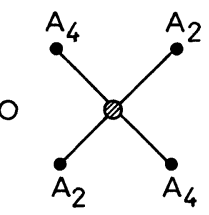

$\otimes$

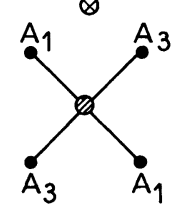

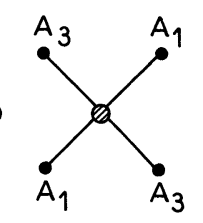

$\otimes$

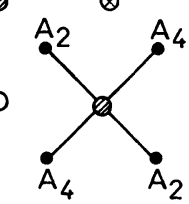

FIG. 2. - Projection sur (100) des cations et des lacunes d'anions de la séquence (1). $\quad \mathrm{A}_{i}$ Lacune d'anion dans le plan $\mathrm{A}_{i}$. $\bigcirc$ Cations de la couche $\alpha$ en site $S_{6}$. $\otimes$ Cations de la couche $\alpha$ en site $C_{2}$. $\emptyset$ Cations de la couche $\beta$ (tous en site $\mathrm{C}_{2}$ ).

[Projection on (100) of the cation and anion vacancy of the sequence (1). $\mathrm{A}_{i}$ anion vacancy in plane $\mathrm{A}_{i}$. $\bigcirc$ Cations in $\alpha$ plane and $\mathrm{S}_{6}$ site. $\otimes$ Cations in $\alpha$ plane and $\mathrm{C}_{2}$ site. $\oslash$ Cations in $\beta$ plane (all in $\mathrm{C}_{2}$ site).]

a) Cisaillement $a / 2\langle 010\rangle$

L'empilement qui résulte de ce déplacement est

$$
\ldots \mathrm{A}_{1} \alpha \mathrm{A}_{2} \beta \mathrm{A}_{3} \uparrow \alpha^{\prime} \mathrm{A}_{3} \beta \mathrm{A}_{2} \beta
$$

si la coupure se fait entre $\mathrm{A}_{3}$ et $\alpha$ ou $\mathrm{A}_{4}$ et $\alpha$

$$
A_{1} \propto A_{2} \beta^{\prime} \uparrow A_{4} \propto A_{3} \beta A_{2} \propto \ldots
$$

lorsqu'elle se produit au niveau d'un plan $\beta$.

Le signe ' $\left(\alpha^{\prime}\right)$ signifie que les cations de la couche $\alpha$ bordant la faute ont vu le nombre (passant de 6 à 4 , 5,7 , ou 8) ou la disposition (de $S_{6}$ ou $C_{2}$ à $6 \mathrm{~A}$, figure 3 ) de leurs premiers voisins modifiés.

Dans le premier cas, de part et d'autre du plan de cations $\alpha^{\prime}$, les deux demi-cristaux sont symétriques et une mâcle est formée. Toutefois deux plans d'anions $\mathrm{A}_{3}$ se retrouvent face à face, ceci devant

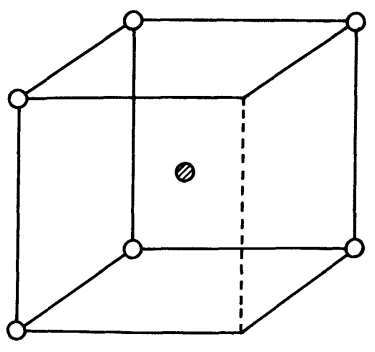

FIG. 3. - Cation en site $6 \mathrm{~A}$.

[Cation in $6 \mathrm{~A}$ site.] 
conduire à une énergie de faute plus élevée que dans le $2^{\mathrm{e}}$ cas. Les cations $\alpha^{\prime}$ ont toujours six premiers voisins anioniques mais disposés comme le montre la figure 3.

b) Cisaillement $a / 2\langle 100\rangle$

La séquence des plans devient dans ce cas

ou

$$
\begin{array}{lllllllllllll}
\ldots & A_{1} & \alpha & A_{2} & \beta & A_{3} & \uparrow & \alpha^{\prime} & A_{1} & \beta & A_{4} & \alpha & \ldots \\
\ldots & A_{1} & \propto & A_{2} & \beta^{\prime} \uparrow & A_{2} & \alpha & A_{1} & \ldots & &
\end{array}
$$

c'est le cisaillement au niveau de $\beta$ qui cette fois produit une mâcle avec le même empilement anionique que précédemment. On doit donc s'attendre à ce que les énergies de faute soient égales.

c) Cisaillement $a / 2\langle 110\rangle$

Cette faute est intéressante car il s'agit d'un déplacement égal au vecteur de Burgers parfait de la structure fluorine.

L'empilement après cisaillement compte tenu du fait que

$$
\begin{array}{ll}
\mathrm{A}_{1} \leftrightarrow \mathrm{A}_{3} & \alpha \leftrightarrow \alpha \\
\mathrm{A}_{2} \leftrightarrow \mathrm{A}_{4} & \beta \leftrightarrow \beta
\end{array}
$$

devient

ou

$$
\mathrm{A}_{1} \propto \mathrm{A}_{2} \quad \beta \quad \mathrm{A}_{3} \alpha^{\downarrow} \mathrm{A}_{2} \quad \beta \quad \mathrm{A}_{3} \propto \mathrm{A}_{4} \quad \beta \ldots
$$

$$
A_{1} \propto A_{2} \beta \downarrow A_{1} \propto A_{2} \beta A_{3} \propto A_{4} \beta
$$

qui sont identiques. Cette faute créée par cisaillement $a / 2\langle 110\rangle$ est identique à celle produite par l'enlèvement de 4 plans successifs de l'empilement du cristal parfait, soit par formation d'une boucle lacunaire intéressant quatre plans. De plus, on peut aisément constater que les cations au niveau de la faute ont le même environnement anionique avant et après la faute; ce qui veut dire que les deux plans d'anions encadrant cette couche de cations ne sont pas modifiés par la faute. L'effet d'un tel cisaillement ne se fera donc sentir qu'en $4^{\mathrm{e}}$ voisin et doit nécessairement conduire à une énergie de faute extrêmement faible (Fig. 4).

4.2 Plan (110). - Chaque plan (110) contient les deux espèces d'ions avec un rapport de stœchiométrie : 1,5. Il s'agit donc d'un plan propice à la formation de boucles de dislocations prismatiques.

La séquence des huit plans (110) peut s'écrire :

$$
\begin{array}{lllllllll}
A_{1} & B_{2} & A_{3} & B_{4} & A_{5} & B_{6} & A_{7} & B_{8}
\end{array}
$$

A et $B$ désignent les deux positions possibles des anions, les couches $\mathrm{A}$ possédant des cations dans des sites $\alpha$ et les couches B des cations dans des sites $\beta$. Par une translation $a / 2\langle 001\rangle$ ou $a / 2\langle 110\rangle$ les plans se déduisent deux à deux comme suit :

$$
A_{1} \leftrightarrow A_{5} \quad B_{2} \leftrightarrow B_{6} \quad A_{3} \leftrightarrow A_{7} \quad B_{4} \leftrightarrow B_{8}
$$

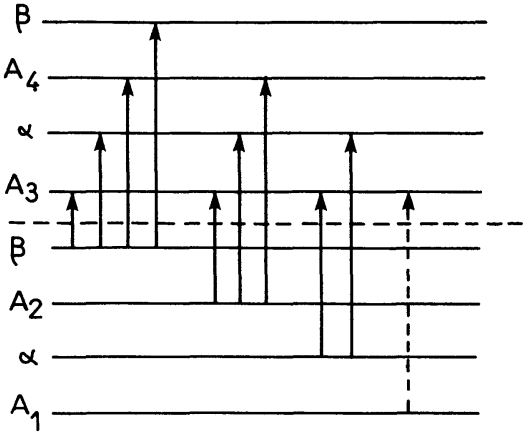

FIG. 4. - Faute $\frac{a}{2}\langle 110\rangle(001)$. En trait plein : interactions non modifiées par le cisaillement. En pointillés : interactions modifiées par le cisaillement.

$\left[\frac{a}{2}\langle 110\rangle(001)\right.$ fault. Full line arrow : interactions un-modified by the shear. Dashed line arrow : interaction modified by the shear.]

Un cisaillement $a / 2\langle 001\rangle$ ou $a / 2\langle 110\rangle$ conduit alors à la séquence suivante :

$$
\begin{array}{llllllllll}
A_{1} & B_{2} & A_{3} & B_{4} & A_{1} & B_{2} & A_{3} & B_{4}
\end{array}
$$

analogue à la faute créée par l'enlèvement de 4 plans (110) successifs.

4.3 Plans (111). - L'empilement comprend 9 plans. Un plan de cations s'insère tous les deux plans d'anions. La distance entre une couche de cations et une couche d'anions étant la moitié de la distance entre deux couches d'anions (égale à $\left.a_{0} \sqrt{3} / 12\right)$. On écrira symboliquement :

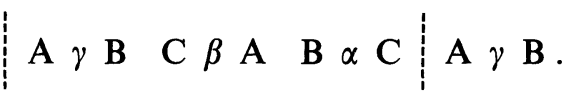

Les lacunes constitutionnelles anioniques forment des motifs réguliers semblables à des $\mathrm{Y}$.

La séquence ci-dessus suggère qu'il y ait le choix entre neuf plans de faute possibles. Ces possibilités se réduisent finalement à 2 . Cisaillement entre deux plans d'anions.

$$
\mathrm{AB} \propto \mathrm{C} \underset{\text { faute } \mathrm{F}_{1}}{\mathrm{~B} \propto \mathrm{C} \quad \mathrm{A}}
$$

ou cisaillement entre un plan d'anions et un plan de cations :

$$
\begin{array}{llll}
B & \mathrm{C} \quad \mathrm{A} & \propto & \mathrm{C} \quad \mathrm{A} \\
& & \\
\text { faute } & \mathrm{F}_{2}
\end{array}
$$

cette dernière faute paraît beaucoup plus difficile à réaliser que la faute $F_{1}$ : en effet anions et cations se retrouvent face à face ce qui bien sûr est très défavorable. Cependant on peut imaginer un processus de synchro shear [8] où les cations se déplacent 
dans une direction différente de la couche d'anions immédiatement supérieure. La séquence devient alors :

$$
\begin{array}{lllllllllll}
\mathbf{A} & \gamma & \mathbf{B} & \mathbf{C} & \uparrow & \mathbf{i} & \mathbf{B} & \mathbf{C} & \beta & \mathrm{A} & \ldots
\end{array}
$$

4.4 RÉSUltats DES CALCUls. - Les valeurs de l'énergie de faute pour les cisaillements décrits précédemment sont résumés dans le tableau suivant

\section{TABLEAU I}

Résultats des calculs d'énergies de faute d'empilement dans $\mathrm{Y}_{2} \mathrm{O}_{3}(\beta / \alpha$ : cisaillement au niveau d'une couche $\beta$ ou $\alpha)$.

[Results of the stacking fault energy calculation in $\mathrm{Y}_{2} \mathrm{O}_{3}(\beta / \alpha$ : shear at $\beta$ or $\alpha$ plane $)$.]

\begin{tabular}{|c|c|c|}
\hline Faute & $\mathrm{EF}\left(\mathrm{mJ} / \mathrm{m}^{2}\right)$ & $\mathrm{EF} / \mu \mathrm{b}$ \\
\hline- & - & - \\
\hline Plan (001) & $\beta / \alpha$ & $\beta / \alpha$ \\
\hline$\frac{a}{4}\langle 100\rangle$ & $4140 / 4831$ & $6 \times 10^{-2} / 7 \times 10^{-2}$ \\
\hline$\frac{a}{2}\langle 100\rangle$ & $2353 / 935$ & $3,4 \times 10^{-2} / 1,3 \times 10^{-2}$ \\
\hline$\frac{a}{4}\langle 010\rangle$ & $4831 / 4140$ & $7 \times 10^{-2} / 6 \times 10^{-2}$ \\
\hline$\frac{a}{2}\langle 010\rangle$ & $935 / 2353$ & $1,3 \times 10^{-2} / 3,4 \times 10^{-2}$ \\
\hline$\frac{a}{4}\langle 110\rangle$ & 842 & $1,2 \times 10^{-2}$ \\
\hline$\frac{a}{2}\langle 110\rangle$ & 79 & $0,1 \times 10^{-2}$ \\
\hline \multicolumn{3}{|l|}{ Plan (110) } \\
\hline$\frac{a}{4}\langle\overline{112}\rangle$ & 1275 & $1,85 \times 10^{-2}$ \\
\hline$\frac{a}{4}\langle 1 \overline{10}\rangle$ & "I & /I \\
\hline$\frac{a}{2}\langle 1 \overline{10}\rangle$ & 2725 & $4 \times 10^{-2}$ \\
\hline$\frac{a}{2}\langle 001\rangle$ & /I & "/ \\
\hline$\frac{a}{4}\langle\overline{11} 1\rangle$ & 5582 & $8 \times 10^{-2}$ \\
\hline \multicolumn{3}{|l|}{ Plan (111) } \\
\hline $\begin{array}{c}\frac{a}{12}\langle 11 \overline{2}\rangle \\
\mathrm{F}_{1}\end{array}$ & 7191 & $1,04 \times 10^{-1}$ \\
\hline$\frac{a}{12}\langle\overline{12} \overline{1}\rangle$ & 7197 & "I \\
\hline $\begin{array}{c}F_{2} \\
F_{2}+S S\end{array}$ & 3477 & $5 \times 10^{-2}$ \\
\hline
\end{tabular}

5. Discussion. - Les calculs d'énergie de faute tels qu'ils sont exposés reposent sur deux approximations :

- le cristal est considéré comme un solide purement ionique.
- Seule la composante électrostatique de l'E.F.E. est prise en considération.

Si la première hypothèse se justifie par la forte ionicité de la liaison $\mathrm{Y}-\mathrm{O}$, la seconde approximation est moins évidente pour les fautes créant des perturbations importantes dans la répartition des sites cationiques. En effet lorsqu'un cation voit modifier le nombre de ses premiers voisins de 2 unités il s'ensuit une forte polarisation électrique qui doit écranter l'interaction électrostatique. Cependant la composante électrostatique étant toujours beaucoup plus importante que les termes de polarisation [8] les valeurs données dans le tableau ci-dessus permettent quand même de comparer l'ordre de grandeur des diverses fautes.

D'une manière générale, les E.F.E. calculées sont plus faibles dans les plans (001) que dans les autres plans considérés, en particulier dans les plans (111) où il n'est pas possible d'analyser ces résultats en termes de dissociation des dislocations. Il est d'ailleurs curieux de noter, dans ces plans (111), la valeur identique de l'énergie électrostatique des fautes $F_{1}$ et $F_{2}$. Cependant une grande différence va intervenir dans les termes de répulsion puisque pour $F_{2}$ anions et cations se trouvent face à face.

Le résultat le plus intéressant des calculs est la valeur exceptionnellement faible pour un cristal ionique de l'E.F.E. correspondant à un cisaillement $b=a / 2\langle 110\rangle(001): \Gamma=80 \mathrm{~mJ} / \mathrm{m}^{2}$ ce qui correspond pour une dislocation coin à une dissociation de l'ordre de $600 \AA$.

De plus, le voisinage de cette faute d'empilement ne variant pas (Fig. 4), la répulsion de Born et les phénomènes de polarisation ne joueront qu'un rôle minime.

D'un point de vue ionique, l'énergie trouvée est donc assez bien représentative de la faute d'empilement. D'autre part, suivant une direction $\langle 110\rangle$ le vecteur de cisaillement, avant de créer la faute $b=a / 2\langle 110\rangle$, passe par la position $b=a / 4\langle 110\rangle$ qui crée une faute d'énergie à peu près dix fois plus forte. La faute d'empilement $b=a / 2\langle 110\rangle$ serait donc une faute stable ce qui n'est pas toujours le cas dans les cristaux ioniques [7]. On peut remarquer que ce vecteur de cisaillement correspond au vecteur de Burgers parfait de la structure fluorine dont est dérivée la structure cubique type $\mathrm{C}$.

L'expérience montre l'importance des plans (001) dans le processus de déformation plastique de $\mathrm{Y}_{2} \mathrm{O}_{3}$ [1]. Ce résultat est surprenant dans la mesure où ces plans ne contiennent pas le vecteur de Burgers le plus court de la structure qui est $b=a / 2\langle 111\rangle$.

Les faibles énergies de faute d'empilement rencontrées dans ces plans, et, en particulier celles créées par $b=a / 2\langle 110\rangle$ peuvent être une explication à ce glissement préférentiel sur (001).

Les observations faites en microscopie électronique à partir de coupes (001) découpées dans un mono- 
cristal d' $\mathrm{Y}_{2} \mathrm{O}_{3}$ déformé tendent à supporter cette hypothèse.

6. Conclusion. - Nous avons calculé les énergies de faute d'empilement dans les plans (100), (110) et
(111) de l'oxyde d'yttrium. Une faute $b=a / 2\langle 110\rangle$ d'énergie très faible $\left(\Gamma=80 \mathrm{~mJ} / \mathrm{m}^{2}\right)$ a été mise en évidence. Ce résultat va dans le sens d'une corrélation étroite entre l'énergie de faute d'empilement et les processus de déformation plastique dans les oxydes.

\section{Bibliographie}

[1] Gaboriaud, R. J., Boisson, M., Grilhé, J., Rapport A.T.P. No 1 SO7 déc. 1976.

[2] Pauling, L., Shappel, M. D., Z. Kristallogr. 75 (1932) 128.

[3] FerT, A., Bull. Soc. Fr, Mineral. Cristallogr. 85 (1962) 267.

[4] Paton, M. G., Maslen, E. N., Acta Crystallogr. 19 (1965) 3, 307.

[5] Foex, M., Traverse, J. P., Bull. Soc. Fr. Mineral. Cristallogr. 89 (1966) 184.
[6] Benson, G. C., Dempsay, E., Proc. Roy. Soc. 266A (1962) 344.

[7] Fontaine, G., J. Phys. \& Chem. Sol. 28 (1967) 2553.

[8] Gaboriaud, R. J., Boisson, M., GrilhÉ, J., J. Phys. C. Solid state Phys. 8 (1975) 3499.

[9] Gaboriaud, R. J., Boisson, M., Grilhé, J., J. Phys. \& Chem. Sol. in press. 